\section{Dr. Fitzcharles, et al reply}

\section{To the Editor:}

We thank Dr. Harth for his comments ${ }^{1}$ on our article examining the Workplace Safety and Insurance Appeals Tribunal (WSIAT) of Ontario, Canada, decisions regarding fibromyalgia (FM) as a compensable condition following workplace injury ${ }^{2}$. In reaching decisions regarding health-related issues, the WSIAT is reliant on input from the medical community that must therefore be reliable, accurate, and reflective of the current state of the medical condition under consideration.

Dr. Harth takes issue with our concern that the WSIAT relied heavily on the opinion of specialists, rheumatologists in particular, in adjudicating the appeals. In our study of decisions, we observed that the WSIAT assigns considerable weight to the opinion of the rheumatologist (74\% of appeals) or advice of a board expert (59\% of appeals), but in only $13 \%$ of appeals was the evidence provided by the family physician noted to be important. Dr. Harth, a "medical consultant to the WSIAT for many years," suggests that family physicians may not be the best-placed healthcare professionals to care for patients with FM because of "GP attitudes" and especially because "23\% thought that patients with FM were malingerers." Because input from experts may guide adjudicators, the lesser importance attributed to the family physician by the WSIAT may have been influenced by advice received.

This bias of the inadequacy of family physicians in issues pertaining to FM has been entrenched in tradition over the last 20 years, buoyed by the claimed ownership of FM by rheumatologists. The family physician is the healthcare professional who is most likely to have both a broad and longitudinal knowledge of the patient, especially regarding health status prior to an alleged work-related incident. The current trend to ascribe an illness such as FM to a workplace event, which in many instances may have been trivial, may be a cunning opportunistic manipulation of a liberal social support system by some persons. The WSIAT denial of one-third of the claims that had reached the level of appeal perhaps suggests that the impression of malingering as stated by primary healthcare providers is not far off the mark for at least some persons labeled as FM. Gervais and colleagues reported that up to a third of persons with FM claiming disability benefits because of alleged FM fail a cognitive effort test, implying exaggeration of symptoms and impairment ${ }^{3}$. Therefore we believe that a diagnosis of FM, a condition with only subjective complaints, may be used by some for reasons of secondary gain. It is for this reason that all medical information provided to adjudicators must be complete and accurate. In most instances of work-related issues and FM, the experts appear on the scene after the fact, with often prolonged time delays between the event and diagnosis of FM. We noted in at least one instance that the consultant rheumatologist definitively pronounced on causality of FM 12 years after the incident. We believe this to be the epitome of "eminence"-based medicine, rather than "evidence-based medicine." The concept that the specialist opinion, with little in-depth knowledge of the patient, rather than that of a family physician should be accepted as a gold standard is redundant.

Dr. Harth belabors the tender point issue, a physical finding without pathological or functional relevance that is fortunately disappearing from the concept of FM. Guided by advice from experts, the WSIAT adjudicators were more likely to accept the diagnosis of FM if a report made mention of tender points. Although tender points were ensconced in the 1990 American College of Rheumatology criteria for FM, we once again emphasize that diagnostic criteria cannot be applied to an individual patient. Dr. Harth correctly states that symptoms of FM or tender points may be faked. It is for exactly this reason that the input from a physician who has seen a patient over a longitudinal period should be considered invaluable in the assessment of patients with FM. By the characteristic of FM persisting over time and manifesting as a polysymptomatic condition, requiring a global biopsychosocial approach to management, ideal care is out of the domain of the busy rheumatologist. In line with current guidelines for the management of persons with FM, the family physician is now acknowledged as the ideal healthcare provider for these patients ${ }^{4,5}$. It therefore follows that this competence will be extended to the assessment of compensation issues. Too often a report of a traumatic event may be used as a convenient tipping point on a background of fragile health, requiring physicians to be vigilant about attributing causation.

Contrary to Dr. Harth's contention, we have made no recommendation for the use of criteria in adjudicating either the diagnosis or causality of FM in an individual patient. The use of criteria to confirm a diagnosis of FM, as was noted in our reading of decisions, is inappropriate, and experts in the pay of the WSIAT should provide responsible advice in this regard. Criteria for diagnosis of FM are of little relevance in the medicolegal evaluation of an individual, but rather emphasis should be placed on functional impairment due to a condition. While it is true that expert opinion is "comforting" for tribunals, it is time to recognize that the family physician has the best composite knowledge of the patient. Our objective in examining the decisions of the WSIAT was to record previous procedures and to provide suggestions for improvement of the process to allow for fair adjudication. The current "epidemic" of FM, mostly in the Western world, the disability reported for this condition, and use of workplace injury cited as a causation should be curtailed, and the medical community is obligated to provide the judiciary with responsible input.

MARY-ANN FITZCHARLES, MB, ChB, Montreal General Hospital; PETER A. STE-MARIE, BA, LLB, Université de Montreal, Faculty of Law; YORAM SHIR, MD, McGill University Health Centre - Alan Edwards Pain Management Unit, Montreal, Quebec, Canada. Address correspondence to Dr. M. Fitzcharles, Montreal General Hospital, 1650 Cedar Avenue, Montreal, Quebec H3G 1A4, Canada;

E-mail: mary-ann.fitzcharles@muhc.mcgill.ca

\section{REFERENCES}

1. Harth M. Work-related physical trauma and fibromyalgia [letter]. J Rheumatol 2013;40:1621-2.

2. Fitzcharles M-A, Ste-Marie PA, Shir Y. A medicolegal analysis of worker appeals for fibromyalgia as a compensable condition following workplace soft-tissue injury. J Rheumatol 2013;40:323-8.

3. Gervais RO, Russell AS, Green P, Allen LM 3rd, Ferrari R, Pieschl SD. Effort testing in patients with fibromyalgia and disability incentives. J Rheumatol 2001;28:1892-9.

4. Sommer C, Häuser W, Burgmer M, Engelhardt R, Gerhold K, Petzke F, et al. Ätiologie und Pathophysiologie der Fibromyalgie-Syndrom [German]. Etiology and pathophysiology of fibromyalgia syndrome. Schmerz 2012;26:259-67.

5. Fitzcharles M-A, Ste-Marie PA, Goldenberg DL, Pereira JX, Abbey $\mathrm{S}$, Choinière $\mathrm{M}$, et al. 2012 Canadian guidelines for the diagnosis and management of fibromyalgia syndrome: Executive summary. Pain Res Manag 2013;18:119-26.

J Rheumatol 2013;40:9; doi:10.3899/jrheum.130446 